\title{
Botulinum Toxin (Type A) as a Minimally Invasive Treatment for Gengival Smile: Case Report
}

\author{
Kethylin Broilo*, Juliana Alves da Silva Ramalho, Priscilla Aparecida Pereira, Karen Muller \\ Ramalho and Susana Morimoto
}

Master in Dentistry Sciences, Ibirapuera University, São Paulo, Brazil

\begin{abstract}
Gingival smile is considered when gingival exposure of $3 \mathrm{~mm}$ or more is visible during smile. Depending on person personality and self-acceptance the gummy smile can lead to aesthetic discomfort while smiling. Depending on the etiology, different treatments may be indicated. However, not all patients are willing to undergo longer or invasive treatments, and in these cases, botulinum toxin - type A (BTA) may be a treatment option. This manuscript reports the case of a patient with a $4 \mathrm{~mm}$ gingival smile. The patient underwent BTA application ( $2 \mathrm{UI} /$ point - total of 2 points) to restore smile harmony. It could be observed that BTA therapy has been proven to be an excellent treatment option in cases of gingival smile caused by muscle hyper-function due to its reversibility, rapid results, efficiency and safety. BTA can be considered a non-invasive method to reduce or eliminate gingival smile in comparison to surgical procedures.
\end{abstract}

\section{Introduction}

Regarding human facial expressions, the smile is the most agreeable and complex in terms of meaning [1]. Therefore, one of the main complaints of the patients happens when the smile is outside the normality patterns, since this situation can influence the self-esteem and the social relationship. Depending on person personality and self-acceptance the gummy smile can lead to aesthetic discomfort while smiling. Therefore, it should be considered that the harmonious smile does not only involve the teeth, from the anatomical and physiological point of view, it also involves the gums, lips and even the eyes have a fundamental participation in the composition of the smile. The contractions of the different facial muscle groups, especially the middle and lower thirds, allow the expression of the smile [1]. Commonly, when smiling the individual exposes between 1 and $3 \mathrm{~mm}$ of gingiva. The exposure of more than $3 \mathrm{~mm}$ of the gum during the smile is known as gingival smile [1]. Mazzuco and Hexsel [1] classified four types of gummy smile: 1) Anterior gummy smile, where more than $3 \mathrm{~mm}$ of gingiva is exposed in the area between the canine teeth involving the action of the levator labii superioris alaeque nasi (LLSAN) muscles; 2) Posterior gummy smile, where more than $3 \mathrm{~mm}$ of gingiva is exposed posterior to the canines, with normal exposure $(<3 \mathrm{~mm})$ in the anterior region involving the action of the zygomatic muscles; 3 ) Mixed gummy smile, with excessive gingival exposure in both (the anterior and posterior regions involving combination of LLSAN and zygomatic muscles; and 4) Asymmetric gummy smile, with excessive or more apparent gingival exposure on one site caused by asymmetric contraction of the Levator labii superioris alaeque nasi (LLSAN) or zygomatic muscles. The
LLSAN muscle is the main target for injection in most studies published. Zygomatic muscles were also described as a muscle targeted for treatment of posterior gummy smile. However, there is no controlled clinical trial comparing the efficacy of techniques [2].

Once the abnormality of the level of gingival exposure is determined during the smile, the establishment of its etiology is essential. Generally, the etiology is multifactorial, and a patient may present one or more factors, mainly related to excessive vertical growth of the maxilla, reduced length of the upper lip, excessive contraction of the upper lip, altered passive eruption, disproportion length/width of the clinical crown of the anterior teeth and hyperfunction of the muscles involved in the smile [3-7].

Treatment options vary according to the etiology of each case, and most treatment options involve a surgical procedure such as gingivoplasty, clinical crown expansion, orthognathic surgery, muscle repositioning surgery or even orthodontic treatment. These treatments are more complex and

*Corresponding author: Kethylin Ferreira Broilo, Master in Dentistry Sciences, Ibirapuera University, São Paulo, Av. Angélica, 321 Cj151, Santa Cecília - SP, Zip Code 01227-000, Brazil

Accepted: November 23, 2020

Published online: November 25, 2020

Citation: Broilo K, Ramalho JAS, Pereira PA, et al. (2020) Botulinum Toxin (Type A) as a Minimally Invasive Treatment for Gengival Smile: Case Report. J Oral Healthc 1(1):11-16 
can generate a greater demand for time and high costs, which may lead to the search for minimally invasive procedures, such as BTA treatment [1-5,7-10].

The use of botulinum toxin for the treatment of gingival smile represents a simple, fast and completely effective method in many cases [1]. The difference is in the results may differ depending on etiological factor, for example, patients who present hyperfunction in the muscles responsible for the elevation of the upper lip during the smile has a more satisfactory result than patients with excessive vertical growth of the maxilla, where the occlusal plane is lower than normal resulting in gingival exposure during a smile [11]. In these cases, for a more satisfactory result, patients would have to undergo orthognathic surgery, however, for some patients, this surgery is not a desire, so botulinum toxin may be a less invasive option.

The use of Botulinum Toxin type A (BTA) for the correction of excessive gingival exposure occurs at points where there is overlapping of muscles: Levator labii superioris alaeque nasi (LLSAN), Levator labii superioris (LLS), zygomaticus minor (ZM) $[2-5,9,10]$ and/or orbicularis oris of the mouth (OO) [4]. Each muscle involved in the upper lip lift has a function during the smile activity. The definition of these points should be established by analysis of the facial mime, regarding the symmetry and amplitude of gingival exposure, asking the patient to smile, followed by palpation during the contraction of specific muscle groups [1].

Onabotulinum toxin $A$, known as BTA, is synthesized by the Gram-positive anaerobic bacterium Clostridium botulin and acts by inhibiting the release of acetylcholine at the neuromuscular junction, preventing muscle contraction and resulting in temporary paralysis of the involved muscle $[3,10]$. There are 7 distinct serotypes of the toxin $(A, B, C 1, d, e, F$ and $G$ ), however, type $A$ is the most frequently subtype used in clinical practice and the most potent [4]. The effect can be perceived after 48 hours, and in 14 days the stability is observed. The neuromuscular transmission and the normal muscle function gradually reestablish and its durability varies according to the patient's metabolism $[4,12]$. When injected, BTA can spread an area of 10 to $30 \mathrm{~mm}$, allowing effective reach $[1,7]$. The most commonly used predetermined site of injection is located laterally to the wing of the nose $[2,7,12]$. When the BTA is injected, it decreases the contraction of the muscles responsible for the elevation of the upper lip, reducing gingival exposure $[1,3,6,7,9,12]$. Also when BTA is applied to the muscles responsible for elevation of the upper lip,

A - Frontal view of the initial smile.

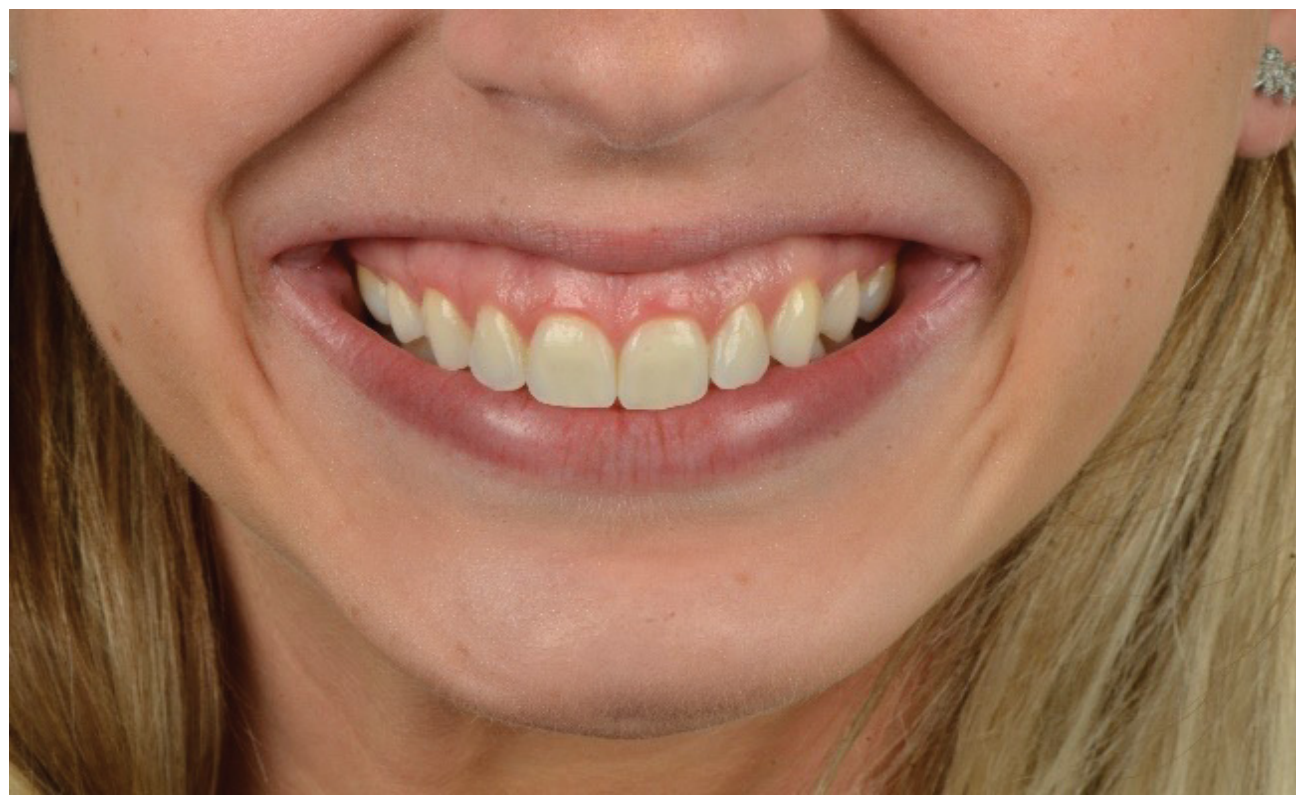

B -Right view of the initial smile

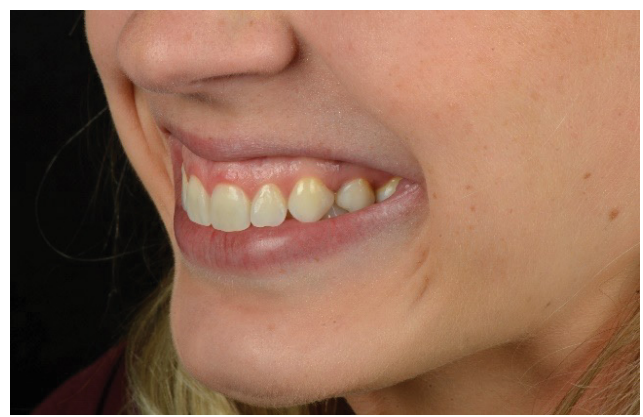

C - Left view of the initial smile

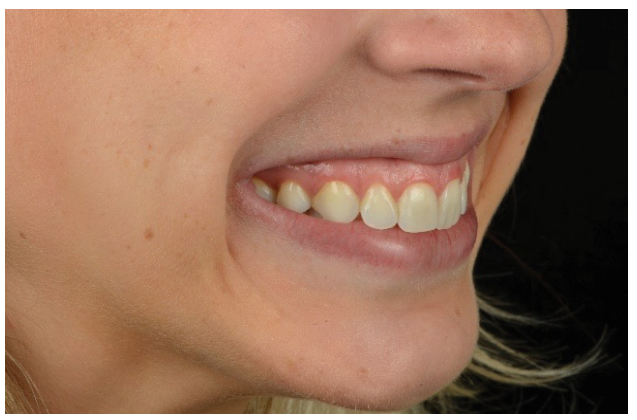

Figure 1: Gingival smile before BTA injection. Fontal and Lateral views of the smile. 
there is inhibition of contraction of the muscle, which allows reducing gingival exposure, and at the same time a smoothing of the nasolabial sulcus may occur [13].

Treatment with BTA has the advantages of being a less invasive procedure. Additionally, it is easy to apply and whose aesthetic improvement is fairly fast and the treatment can also serve as an adjunct to surgical procedures $[6,7,10]$. This case report describes the protocol to reduce gingival smile through BTA application.

\section{Case Presentation}

A female patient, 27-years-old, was really unsatisfied with the aesthetics of her smile due to excessive gingival smile, classified as mixed gummy smile, with excessive gingival exposure in the anterior and posterior regions. A careful anamnesis was carried out, with questions about his current health state and about possible allergies. Patient had no contraindication to the use of BTA such as pregnancy, neuromuscu- lar diseases or use of specific medications [1]. Extra and intraoral examination was performed, as well as photographs were taken (Figure 1). Clinical examination revealed $4 \mathrm{~mm}$ of gingival exposure during smile (Figure 2). The points considered for gingival measurement is indicated in Figure 2. The smile could be classified as mixed gingival smile, once excessive gum exposure was present in both anterior and posterior region (Figure 1 and Figure 2). As patient does not present skeletal discrepancies, gingival smile was attributed to the hyper-functional upper lip elevator muscles. Patient was not willing to undergo surgical procedures for aesthetic purposes. As a non-invasive alternative treatment, it was proposed the injection of botulinum toxin - type A (BTA) with the aim to reduce or eliminate gingival exposure during smile. Before any clinical intervention, patient signed the Informed Consent Term specifying all the procedures to be performed, such as risks, possible asymmetrical results and authorization of using her image. The protocol of BTA application is described in Table 1 (Figure 3).

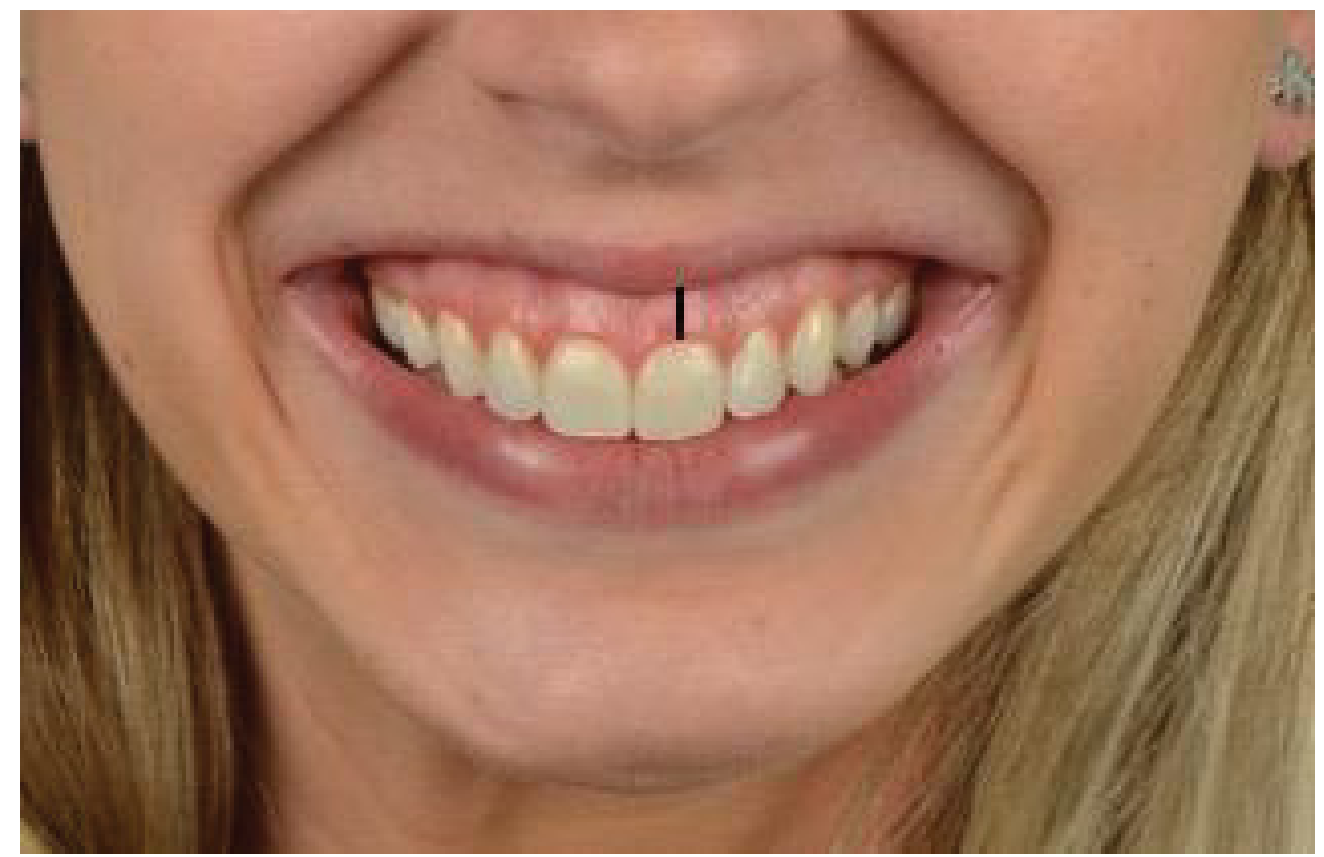

Figure 2: Measurement of gingival display. Both end of black line showed the points from which measurements were taken.

Table 1: Protocol of BTA application.

1. Hygiene of the face with $70 \%$ alcohol - A cotton soaked in alcohol $70 \%$ was used for skin cleansing.

2. Markings of the injection points with white pencil (Vult ${ }^{\circ}$ ) or similar (Figure 3 ). The Point was considered $2 \mathrm{~mm}$ lateral to the alarfacial groove at the level of the nasal passage, at both sides, to relax the Levator labii superioris alaeque nasi (LLSAN) muscle.

3. A bottle of 100 units (U) Botulinum Toxin type $A$ (Botox ${ }^{\circ}$ ) was diluted with the aid of a $3 \mathrm{ml}$ syringe together with a disposable 22 G1 hypodermic needle in $1 \mathrm{ml}$ of $0.9 \%$ saline sterile solution immediately before injection, following the recommendation guidelines on dilution and storage.

4. Prior to injection, the region was cooled with ice for 5 seconds to promote temporary analgesia.

5. It was applied $2 \mathrm{U}$ of BTA in each point in the subcutaneous tissue through a BD Insulin syringe ultra-fine TM (BM Medical Diabetes Care, Holdrege, USA) $30 \mathrm{U}$. with $8 \mathrm{~mm} \times 30 \mathrm{G}$ needle.

6. Patient was instructed with the recommendation: To maintain elevated head for $4 \mathrm{~h}$; do not compress the region; not performing physical activities for 24 hours.

7. Photographs of the final result were taken 20 days after the injection. 


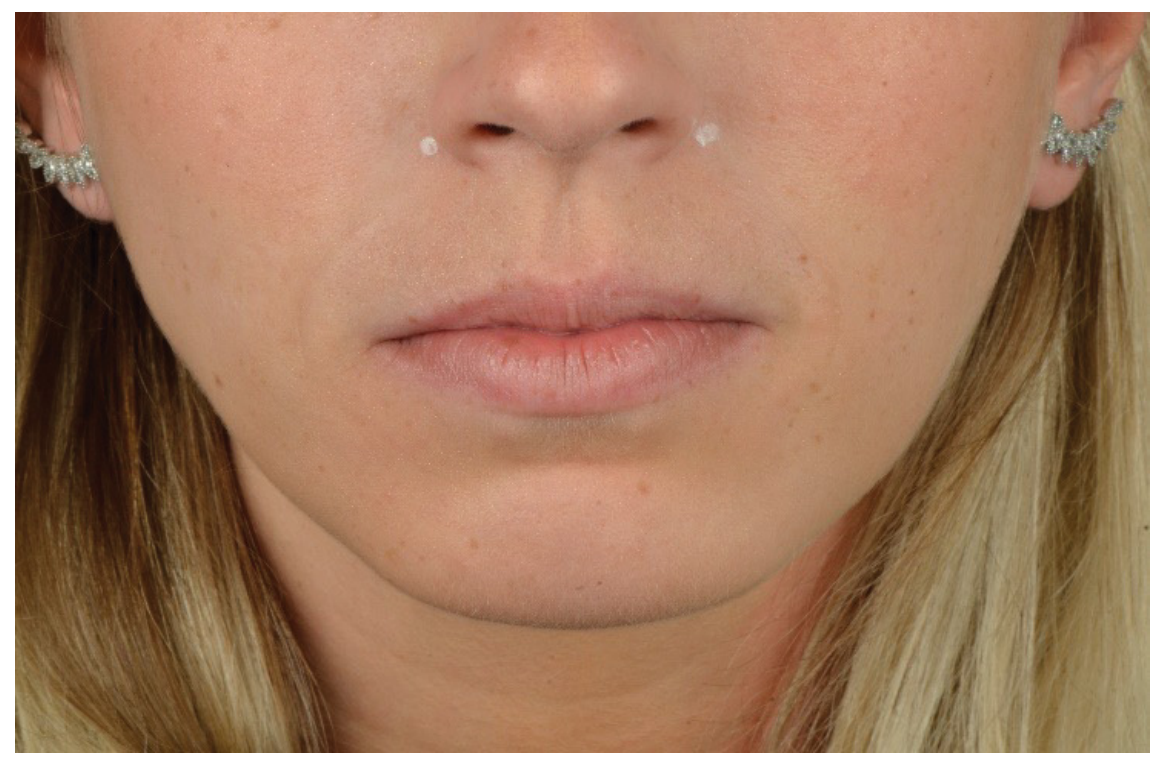

Figure 3: Markings of the injection points with white pencil. Point was considered $2 \mathrm{~mm}$ lateral to the alar-facial groove at the level of the nasal passage, at both sides.

\section{A - Frontal view of the final smile}

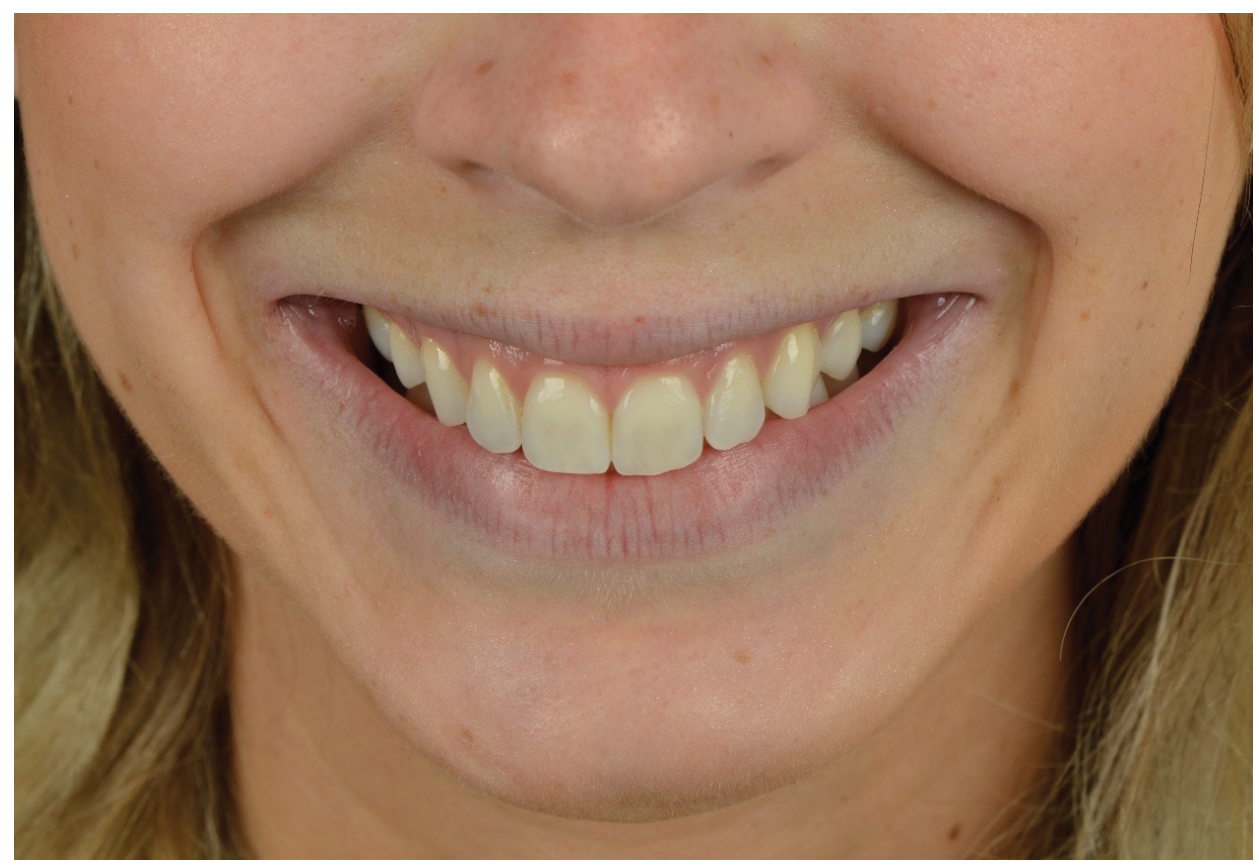

B -Left view of the initial smile

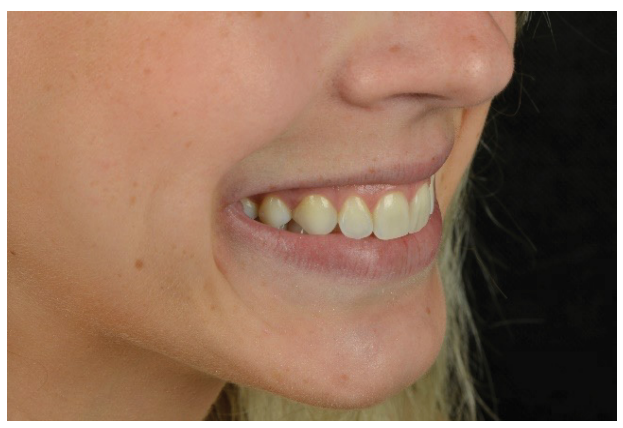

C - Right view of the initial smile

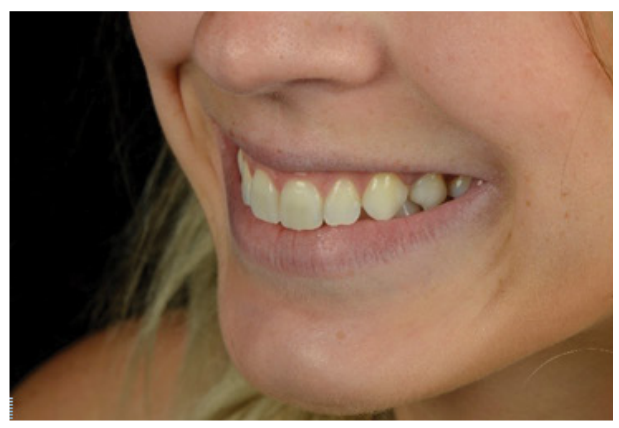

Figure 4: Final result after 20 days of injection. 
A- Initial smile

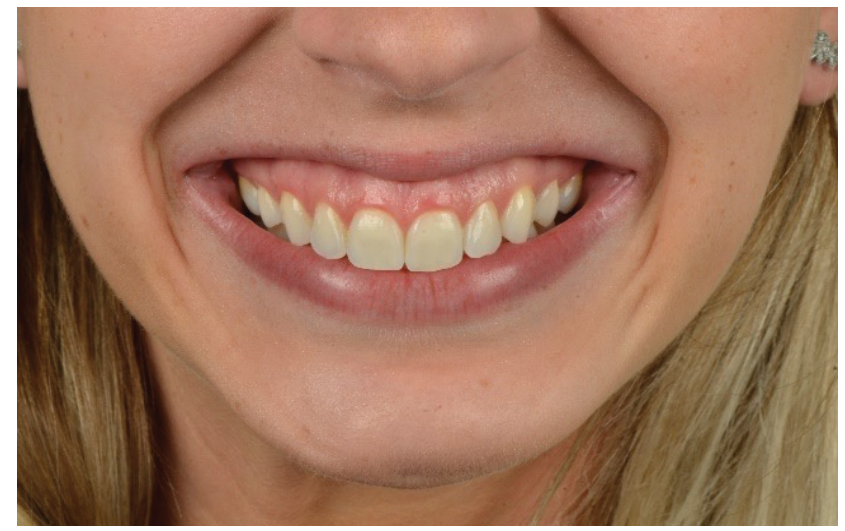

B - Final smile

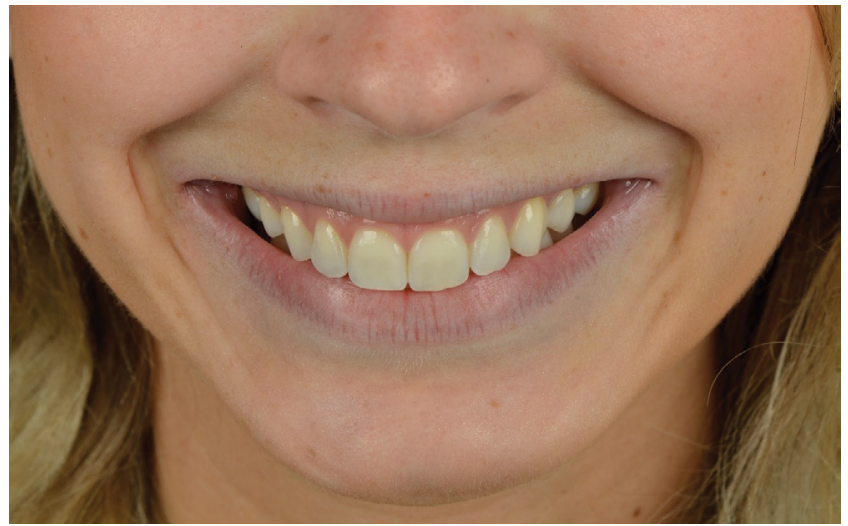

Figure 5: Initial and Final aspect of smile.

The clinical effects began 2 days after application, and the maximum visible effects occurred after 14 days. After 20 days the photographs and the measurements of the smile were carried out (Figure 4). A decreased of $2 \mathrm{~mm}$ was observed in the exposed gingiva during smile. No side effects have been reported such as bruising, edema or elongation of the upper lip. In the present report, the result achieved was satisfactory in reduction of gingival smile, while still promoting smooth facial lines of the smile, as can be observed in the nasolabial sulcus, adjacent to the nostrils (Figure 4). The clinical outcome remained for about 4 months. The comparison between smile before and after treatment can be observed in Figure 5 .

\section{Discussion}

The relevance of the smile is a relevant theme considering that it represents a very important aspect for socialization $[1,9]$. It is a fact that the aesthetics requirement of patients is increasing over time. Therefore, planning should take into account not only predetermined and standardized aspects, but also the relationship of the smile with the patient's face. It is necessary that the professional shows common sense to promote a harmonious and balanced face, requiring, therefore, a diagnosis and planning with a multidisciplinary knowledge [8].

The dissatisfaction with a gingival smile displays vary between cultures and ethnic conventions. Different sizes of gingival exposure were mentioned in literature, in most cases it is conceptualized by exposing more than $3 \mathrm{~mm}$ of gingival tissue $[1,6]$. However, it was considered a gingival smile when the exposure was greater than 2-3 $\mathrm{mm}$, and was found most often in women [9].

There are several etiological factors of the gingival smile such as vertical maxillary excess $[1-5,7,12]$, late passive eruption $[4,5,7]$, hyperfunction of muscles involved in the smile $[6,7,12]$; reduced length of clinical crown of teeth [6] reduced upper lip length, excessive contraction of the upper lip [7] and anterior dentoalveolar extrusion. These etiologies may occur singly or in combination, and determine the type of treatment to be employed $[6,7]$.
For the gingival smile treatment, several modalities are proposed such as surgical, non-surgical or a combination of both. The association of BTA injection with surgical procedures such as Lefort 1 osteotomy, gingival repositioning surgery and gingivectomy may be applied [14].

For patients who are not willing to undergo invasive surgical procedures, BTA has become an excellent alternative in these cases. Thus, according to this study and the literature, the BTA seems to be an alternative, used to improve cases of gingival smile determined by muscular etiology, when there is a hyper-contracting of the upper lip lifting muscles. BTA effect is time dependent and will wear off over 3-4 months.

The local points of BTA application for the gingival smile vary among the muscles in the literature. However, they converge most often to the overlapping of three muscles (Levator labii superioris, Levator labii superioris alaeque nasi and zygomaticus minor), regardless of gender, age or ethnicity $[4,7]$.

In Sucupira \& Abramovitz [9] and Suber, et al. [8], BTA injections were applied to the muscles responsible for labial elevation, and concluded that gingival smile treatment proved to be safe and effective, minimally invasive and that can significantly improve smile aesthetics and patient satisfaction. These results are in accordance with Polo [4,5], as well as those obtained by Hwang, et al. $[7,10]$ and Sandler, et al. [10]. The clinical case presented in this study also showed favorable results within reduction of gingival exposure in 2 $\mathrm{mm}$, besides promoting a smoothness to the facial lines, as observed in the nasogenian sulcus.

Mazzuco \& Hexsel [1] strongly indicated the use of BTA in all cases of oral muscular hyperactivity. Thus, the expected and programmed relapse in BTA treatments seems to be more of an advantage because of its non-definitive character than a disadvantage due to the need for reapplication.

The Injection of BTA into the upper lip lift muscle proved to be an effective method, offering safe injection without complications. However, the study of Sandler, et al. [9] presented a report of a patient with difficulty to eat and in the pronunciation of some words in the first week, which was not observed with the patient described in our case report. Other 
side effects are described in literature: The treatment results may require weeks to stabilize and may begin with reports of a slight fall of the upper lip, which becomes unpleasant for some patients; among other reports associated with the fact that injections cause discomfort. Asymmetrical results may occur and still lead to a "joker smile", which results from excessive pulling up and to the side of the larger zygomatic muscles, due to the reduced tonicity of the lifting muscles $[14,15]$.

Clinical effects occur within 2 to 10 days after injection, and the maximum visible effect occurs after 14 days of injection $[4,7]$. According literature this effect lasts approximately 3 to 6 months $[1,4,12]$. However, in advanced and severe cases, such as that presented by Indra, et al. [3], it is possible to have an earlier recurrence ( 3 months). The application of BTA was effective in the present case of gingival smile after 20 days, remaining the clinical result for 4 months. After recurrence of gingival exposure, toxin reapplication is required.

\section{Conclusion}

The application of BTA is a therapeutic technique that is fast and effective in reducing the gingival smile. It promotes a better positioning of the upper lip, improving the aesthetics of the smile, but it is a treatment with transient effects that require reapplications every 4-6 months in average.

\section{Ethical Approval}

All procedures followed were in accordance with the ethical standards of the responsible committee on human experimentation (institutional and national) and with the Helsinki Declaration of 1975, as revised in 2008.

\section{Consent}

Informed consent was obtained from all patients for being included in the study.

\section{Conflicts of Interest}

All of the authors do not have any conflict of interest in the data collection, interpretation of the results, and writing of the article.

\section{Authors' Contribution}

All of the authors contributed to the formation of the article.

\section{References}

1. Mazzuco R, Hexsel D (2010) Gummy smile and botulinum toxin: A new approach based on the gingival exposure area. J Am Acad Dermatol 63: 1042-1051.

2. Onurcem Duruel, Emel Tuğba Ataman-Duruel, Ezel Berker, et al. (2019) Treatment of various types of gummy smile with botulinum Toxin-A. J Craniofac Surg 30: 876-878.

3. Adarsh S Indra, PP Biswas, VT Vineet, et al. (2011) Botox as an adjunct to orthognathic surgery for a case of severe vertical maxillary excess. J Maxillofac Oral Surg 10: 266-270.

4. Polo $M(2005)$ Botulinum toxin type $A$ in the treatment of excessive gingival display. Am J Orthod Dentofacial Orthop 127: 214-218.

5. Polo M (2008) Botulinum toxin type A (Botox) for the neuromuscular correction of excessive gingival display on smiling (gummy smile). Am J Orthod Dentofacial Orthop 133: 195-203.

6. Alessandro Mangano, Alberto Mangano (2012) Current strategies in the treatment of gummy smile using botulinum toxin type A. Plast Reconstr Surg 129: 1015e.

7. Woo-Sang Hwang, Mi-Sun Hur, Kyung-Seok Hu, et al. (2009) Surface anatomy of the lip elevator muscles for the treatment of gummy smile using botulinum toxin. Angle Orthod 79: 70-77.

8. Jessica S Suber, Trish P Dinh, Melanie D Prince, et al. (2014) OnabotulinumtoxinA for the treatment of a "gummy smile". Aesthet Surg J 34: 432-437.

9. Sucupira E, Abramovitz A (2012) A simplified method for smile enhancement: Botulinum toxin injection for gummy smile. Plast Reconstr Surg 130: 726-728.

10. Sandler P, Alsayer F, Davies S (2007) Botox: A possible new treatment for gummy smile. Virtual J Orthod 20: 30-34.

11. Slberberg N, Goldstein M, Smidt A (2009) Excessive gingival display - Etiology, diagnosis, and treatment modalities. Quintessence Int 40: 809-818.

12. Jaspers GW, Pijpe J, Jansma J (2011) The use of botulinum toxin type A in cosmetic facial procedures. Int J Oral Maxillofac Surg 40: 127-133.

13. Ishida $Y$, Ono T (2017) Nonsurgical treatment of an adult with a skeletal Class II gummy smile using zygomatic temporary anchorage devices and improved superelastic nickel-titanium alloy wires. Am J Orthod Dentofacial Orthop 152: 693-705.

14. Mostafa D (2018) A successful management of sever gummy smile using gingivectomy and botulinum toxin injection: A case report. Int J Surg Case Rep 42: 169-174.

15. Almasri M (2015) Surgical techniques to improve the smile. In: A textbook of advanced oral and maxillofacial surgery.

DOI: $10.36959 / 704 / 675$

Copyright: (c) 2020 Broilo K, et al. This is an open-access article distributed under the terms of the Creative Commons Attribution License, which permits unrestricted use, distribution, and reproduction in any medium, provided the original author and source are credited. 